\title{
MULTIPLE POSITIVE SOLUTIONS FOR QUASILINEAR ELLIPTIC PROBLEMS WITH SIGN-CHANGING NONLINEARITIES
}

\author{
JULIÁN FERNÁNDEZ BONDER
}

Received 19 May 2003

Using variational arguments, we prove some nonexistence and multiplicity results for positive solutions of a system of $p$-Laplace equations of gradient form. Then we study a $p$-Laplace-type problem with nonlinear boundary conditions.

\section{Introduction}

In a recent paper, [7], the authors studied the existence, multiplicity, and nonexistence of positive classical solutions of the semilinear elliptic boundary value problem

$$
\begin{aligned}
-\Delta u & =\lambda f(u) \quad \text { in } \Omega, \\
u & =0 \quad \text { on } \partial \Omega,
\end{aligned}
$$

where $\Omega$ is a smooth bounded domain in $\mathbb{R}^{N}, N \geq 1, \lambda>0$ is a parameter, and $f$ is a $C^{1}$ sign-changing sublinear function.

They showed using sub-super solutions arguments and recent results from semipositone problems that there are $\underline{\lambda}$ and $\bar{\lambda}$ such that (1.1) has no positive solution for $\lambda<\underline{\lambda}$ and at least two positive solutions for $\lambda \geq \bar{\lambda}$.

More recently, in [8], the author extends these results to the quasilinear problem

$$
\begin{aligned}
-\Delta_{p} u & =\lambda f(x, u) \quad \text { in } \Omega, \\
u & =0 \quad \text { on } \partial \Omega,
\end{aligned}
$$

where $\Delta_{p} u=\operatorname{div}\left(|\nabla u|^{p-2} \nabla u\right)$ is the $p$-Laplacian, $1<p<\infty, \lambda>0$, and $f$ is a sign-changing Carathéodory function on $\Omega \times[0, \infty)$.

The method in [8] is variational and allowed the author to substantially relax the assumptions on $f$. More precisely, these assumptions are

$\left(\mathrm{H}_{1}\right) f(x, 0)=0,|f(x, t)| \leq C|t|^{p-1}$,

$\left(\mathrm{H}_{2}\right)$ there exists $\delta>0$ such that $F(x, t) \leq 0$ for $0 \leq t \leq \delta$, 
1048 Quasilinear problems with sign-changing nonlinearities

$\left(\mathrm{H}_{3}\right)$ there exists $t_{0}>0$ such that $F\left(x, t_{0}\right)>0$,

$\left(\mathrm{H}_{4}\right) \lim \sup _{t \rightarrow \infty} F(x, t) / t^{p} \leq 0$ uniformly in $x$, where $F(x, t)=\int_{0}^{t} f(x, s) d s$.

The purpose of this article is twofold. Applying variational methods, we first extend the results in [8] to quasilinear elliptic systems of the form

$$
\begin{aligned}
-\Delta_{p} u & =\lambda F_{u}(x, u, v) \quad \text { in } \Omega, \\
-\Delta_{q} v & =\lambda F_{v}(x, u, v) \quad \text { in } \Omega, \\
u & =v=0 \quad \text { on } \partial \Omega,
\end{aligned}
$$

where $\left(F_{u}, F_{v}\right)$ stands for the gradient of a given potential $F$, and second, we want to see to what extent these variational techniques can be adapted to deal with the nonlinear boundary condition case

$$
\begin{array}{cc}
-\Delta_{p} u+|u|^{p-2} u=0 & \text { in } \Omega, \\
|\nabla u|^{p-2} \frac{\partial u}{\partial v}=\lambda g(x, u) & \text { on } \partial \Omega,
\end{array}
$$

where $\partial / \partial \nu$ is the outer unit normal derivative.

Systems of the form (1.3) are usually called gradient systems and have been widely studied in the past. See, for example, [2] for a comprehensive analysis of such systems. This gradient structure allows us to treat (1.3) variationally. Other kinds of elliptic systems that can be treated variationally are the so-called Hamiltonian systems, see [3].

However, as far as we know, all the results for (1.3) assume, to begin with, that $F_{u}, F_{v} \geq 0$ for $u, v \geq 0$.

For problem (1.4), in a previous paper, [4], the authors studied the problem where the nonlinearity $g$ was assumed to be of power type, that is, essentially the case $g(x, t)=$ $|t|^{q-2} t$ was considered, so again $g(x, t) \geq 0$ for $t \geq 0$.

The main results of this paper can be formulated as follows.

Under hypotheses similar to $\left(\mathrm{H}_{1}\right)-\left(\mathrm{H}_{4}\right)$, there exists $0<\underline{\lambda}<\bar{\lambda}$ such that if $0<\lambda<\underline{\lambda}$ problem (1.3) (or problem (1.4)) has no positive solution and if $\lambda>\bar{\lambda}$ problem (1.3) (or problem (1.4)) has, at least, two positive solutions.

The rest of the paper is organized as follows: in Section 2 we deal with problem (1.3) and in Section 3 with (1.4).

\section{Gradient systems}

In this section, we deal with problem (1.3). First, we prove the nonexistence result. To this end, we assume that $F(x, u, v)$ is a Carathéodory function on $\Omega \times[0, \infty) \times[0, \infty), F(x, \cdot, \cdot)$ is $C^{1}$ for a.e. $x \in \Omega$, and $F_{u}, F_{v}$ are also Carathéodory functions satisfying

$$
\begin{gathered}
F(x, 0,0)=F_{u}(x, 0,0)=F_{v}(x, 0,0)=0, \\
\left|u F_{u}(x, u, v)+v F_{v}(x, u, v)\right| \leq C\left(u^{p}+v^{q}\right), \\
|F(x, u, v)| \leq C\left(u^{p}+v^{q}\right),
\end{gathered}
$$

for some constant $C>0$. 
We have the following theorem.

Theorem 2.1. Assume (2.1) holds. Then, there is a $\underline{\lambda}$ such that (1.3) has no positive solution for $\lambda<\underline{\lambda}$.

For the proof we need the following observation. We denote by $\lambda_{r}$ the best constant in the Sobolev embedding $W_{0}^{1, r}(\Omega) \hookrightarrow L^{r}(\Omega)$. We have

$$
\begin{array}{ll}
\lambda_{p} \int_{\Omega}|u|^{p} d x \leq \int_{\Omega}|\nabla u|^{p} d x \quad \text { for } u \in W_{0}^{1, p}(\Omega), \\
\lambda_{q} \int_{\Omega}|v|^{q} d x \leq \int_{\Omega}|\nabla v|^{q} d x \quad \text { for } v \in W_{0}^{1, q}(\Omega),
\end{array}
$$

so if we denote $\lambda_{p, q}=\min \left\{\lambda_{p}, \lambda_{q}\right\}$, we obtain

$$
0<\lambda_{p, q} \leq \frac{\int_{\Omega}|\nabla u|^{p}+|\nabla v|^{q} d x}{\int_{\Omega}|u|^{p}+|v|^{q} d x} \quad \text { for } u \in W_{0}^{1, p}(\Omega), v \in W_{0}^{1, q}(\Omega)
$$

and, moreover, one can easily see that $\lambda_{p, q}$ is optimal.

Proof of Theorem 2.1. If (1.3) has a positive solution $(u, v)$, multiplying the first equation of (1.3) by $u$, the second by $v$, and integrating by parts and adding up, we get

$$
\int_{\Omega}|\nabla u|^{p}+|\nabla v|^{q} d x=\lambda \int_{\Omega} F_{u}(x, u, v) u+F_{v}(x, u, v) v d x
$$

Thus, using (2.1), we obtain

$$
\int_{\Omega}|\nabla u|^{p}+|\nabla v|^{q} d x \leq \lambda C \int_{\Omega}|u|^{p}+|v|^{q} d x
$$

and hence $\lambda \geq \lambda_{p, q} / C$ by (2.3), proving Theorem 2.1.

Now, we prove the multiplicity result. To this end, along with (2.1), we also have to assume that

$\left(\mathrm{F}_{1}\right)$ there exists $\delta>0$ such that $F(x, u, v) \leq 0$ for $|u|^{p}+|v|^{q} \leq \delta$,

$\left(\mathrm{F}_{2}\right)$ there exists $t_{0}, s_{0}>0$ such that $F\left(x, t_{0}, s_{0}\right)>0$,

$\left(\mathrm{F}_{3}\right) \lim \sup _{|(u, v)| \rightarrow \infty} F(x, u, v) /\left(u^{p}+v^{q}\right) \leq 0$ uniformly in $x$.

Under these assumptions, we have the following theorem.

Theorem 2.2. Under the assumptions (2.1), $\left(F_{1}\right),\left(F_{2}\right)$, and $\left(F_{3}\right)$, there is a $\bar{\lambda}$ such that (1.3) has at least two positive solutions $\left(u_{1}, v_{1}\right),\left(u_{2}, v_{2}\right)$ for $\lambda \geq \bar{\lambda}$.

For the proof of Theorem 2.2, we use critical point theory. Set $F(x, u, v)=0$ for $u, v<0$, and consider the $C^{1}$ functional

$$
\mathscr{F}_{\lambda}(u, v)=\int_{\Omega} \frac{|\nabla u|^{p}}{p}+\frac{|\nabla v|^{q}}{q}-\lambda F(x, u, v) d x, \quad(u, v) \in W_{0}^{1, p}(\Omega) \times W_{0}^{1, q}(\Omega) .
$$


1050 Quasilinear problems with sign-changing nonlinearities

Observe that if $(u, v)$ is a critical point of $\mathscr{F}_{\lambda}$, denoting by $u^{-}$and $v^{-}$the negative parts of $u$ and $v$, respectively,

$$
\begin{aligned}
0= & \left(\mathscr{F}_{\lambda}^{\prime}(u, v),\left(u^{-}, v^{-}\right)\right) \\
= & \int_{\Omega}|\nabla u|^{p-2} \nabla u \cdot \nabla u^{-}+|\nabla v|^{q-2} \nabla v \cdot v^{-} \\
& \quad-\lambda\left(F_{u}(x, u, v) u^{-}+F_{v}(x, u, v) v^{-}\right) d x \\
= & \left\|u^{-}\right\|_{W_{0}^{1, p}(\Omega)}^{p}+\left\|v^{-}\right\|_{W_{0}^{1, q}(\Omega)}^{q},
\end{aligned}
$$

hence we have that $u, v \geq 0$. Furthermore, by [10], $u, v \in C^{1, \alpha}(\Omega)$ and so, by Harnack inequality (see [11]), it follows that either $u, v>0$ or $u \equiv v \equiv 0$. Therefore, nontrivial critical points of $\mathscr{F}_{\lambda}$ are positive solutions of (1.4).

By $\left(\mathrm{F}_{3}\right)$ and $(2.1)$, there is a constant $C_{\lambda}>0$ such that

$$
\lambda F(x, u, v) \leq \frac{\lambda_{p, q}}{2}\left(\frac{|u|^{p}}{p}+\frac{|v|^{q}}{q}\right)+C_{\lambda}
$$

and hence

$$
\begin{aligned}
\mathscr{F}_{\lambda}(u) & \geq \int_{\Omega} \frac{|\nabla u|^{p}}{p}+\frac{|\nabla v|^{q}}{q}-\frac{\lambda_{p, q}}{2}\left(\frac{|u|^{p}}{p}+\frac{|v|^{q}}{q}\right)-C_{\lambda} d x \\
& \geq \frac{1}{2 p}\|u\|_{W_{0}^{1, p}(\Omega)}^{p}+\frac{1}{2 q}\|v\|_{W_{0}^{1, q}(\Omega)}^{q}-C_{\lambda}|\Omega|_{N},
\end{aligned}
$$

where $|\cdot|_{d}$ denotes the $d$-dimensional Lebesgue measure in $\mathbb{R}^{N}$, so $\mathscr{F}_{\lambda}$ is bounded from below and coercive.

Therefore, as $\mathscr{F}_{\lambda}$ is weakly lower semicontinuous, we obtain a global minimizer $\left(u_{1}\right.$, $\left.v_{1}\right)$. We show that, if $\lambda$ is big enough, this minimizer is nontrivial.

Lemma 2.3. There is a $\bar{\lambda}$ such that $\inf \mathscr{F}_{\lambda}<0$, and hence $\left(u_{1}, v_{1}\right) \neq(0,0)$, for $\lambda \geq \bar{\lambda}$.

Proof. We consider a sufficiently large compact subset $\Omega^{\prime}$ of $\Omega$ and take functions $u_{0} \in$ $W_{0}^{1, p}(\Omega), v_{0} \in W_{0}^{1, q}(\Omega)$ such that $u_{0}(x)=t_{0}$ on $\Omega^{\prime}, 0 \leq u_{0}(x) \leq t_{0}$ on $\Omega \backslash \Omega^{\prime}, v_{0}(x)=s_{0}$ on $\Omega^{\prime}, 0 \leq v_{0}(x) \leq s_{0}$ on $\Omega \backslash \Omega^{\prime}$, where $t_{0}, s_{0}$ are as in $\left(\mathrm{F}_{2}\right)$.

Then, we obtain

$$
\int_{\Omega} F\left(x, u_{0}, v_{0}\right) d x \geq \int_{\Omega^{\prime}} F\left(x, t_{0}, s_{0}\right) d x-C\left(t_{0}^{p}+s_{0}^{q}\right)\left|\Omega \backslash \Omega^{\prime}\right|_{N}>0,
$$

if $\Omega^{\prime}$ is big enough. Hence, $\mathscr{F}_{\lambda}\left(u_{0}, v_{0}\right)<0$ for $\lambda$ large enough.

We will obtain a critical point $\left(u_{2}, v_{2}\right)$ with $\mathscr{F}_{\lambda}\left(u_{2}, v_{2}\right)>0$ via the mountain pass lemma, which would complete the proof since $\mathscr{F}_{\lambda}\left(u_{2}, v_{2}\right)>0>\mathscr{F}_{\lambda}\left(u_{1}, v_{1}\right)$. 
LEMma 2.4. The origin is a strict local minimizer of $\mathscr{F}_{\lambda}$.

Proof. Let $U_{(u, v)}=\left\{x \in \Omega:|u(x)|^{p}+|v(x)|^{q}>\delta\right\}$. By $\left(\mathrm{F}_{1}\right), F(x, u(x), v(x)) \leq 0$ on $\Omega \backslash$ $U_{(u, v)}$, so

$$
\mathscr{F}_{\lambda}(u, v) \geq \frac{1}{p}\|u\|_{W_{0}^{1, p}(\Omega)}^{p}+\frac{1}{q}\|v\|_{W_{0}^{1, q}(\Omega)}^{q}-\lambda \int_{U_{(u, v)}} F(x, u, v) d x
$$

By (2.1), Hölder's inequality, and Sobolev embedding,

$$
\begin{aligned}
\int_{U_{(u, v)}} F(x, u, v) d x & \leq C \int_{U_{(u, v)}} u^{p}+v^{q} d x \\
& \leq C\left(\left|U_{(u, v)}\right|_{N}^{1-p / r}\|u\|_{W_{0}^{1, p}(\Omega)}^{p}+\left|U_{(u, v)}\right|_{N}^{1-q / s}\|v\|_{W_{0}^{1, q}(\Omega)}^{q}\right),
\end{aligned}
$$

where $r=N p /(N-p)$ if $p<N$ and $r>p$ if $p \geq N$, and $s=N q /(N-q)$ if $q<N$ and $s>q$ if $q \geq N$. So, in order to finish the proof we need to show that $\left|U_{(u, v)}\right|_{N} \rightarrow 0$ as $\|u\|_{W_{0}^{1, p}(\Omega)}+\|v\|_{W_{0}^{1, q}(\Omega)} \rightarrow 0$.

Now,

$$
\|u\|_{W_{0}^{1, p}(\Omega)}^{p}+\|v\|_{W_{0}^{1, q}(\Omega)}^{q} \geq \lambda_{p, q} \int_{\Omega} u^{p}+v^{q} d x \geq \lambda_{p, q} \int_{U_{(u, v)}} u^{p}+v^{q} d x \geq \lambda_{p, q} \delta\left|U_{(u, v)}\right|_{N},
$$

as we wanted to show.

Now, we are in position to finish the proof of Theorem 2.2.

Proof of Theorem 2.2. As $\mathscr{F}_{\lambda}$ is coercive, every Palais-Smale sequence is bounded and hence contains a convergent subsequence as usual. Now, the mountain pass lemma gives a critical point $\left(u_{2}, v_{2}\right)$ of $\mathscr{F}_{\lambda}$ at the level

$$
c:=\inf _{\gamma \in \Gamma(u, v) \in \gamma([0,1])} \max _{\lambda} \mathscr{F}_{\lambda}(u, v)>0
$$

where $\Gamma=\left\{\gamma \in C\left([0,1], W_{0}^{1, p}(\Omega) \times W_{0}^{1, q}(\Omega)\right): \gamma(0)=0, \gamma(1)=\left(u_{1}, v_{1}\right)\right\}$ is the class of paths joining the origin to $\left(u_{1}, v_{1}\right)$ (see [9]).

\section{The nonlinear boundary condition case}

In this section, we deal with the nonlinear boundary condition case, problem (1.4). The main ideas and structures of the proofs are the same as in the previous section, so we only sketch them and stress the differences between the two cases.

We begin with the nonexistence result. To this end, we assume that $g$ is a Carathéodory function on $\partial \Omega \times[0, \infty)$ satisfying

$$
g(x, 0)=0, \quad-c t^{r-1} \leq g(x, t) \leq C t^{p-1}
$$

for some $1 \leq r \leq p$ and some constants $C, c>0$. 
1052 Quasilinear problems with sign-changing nonlinearities

We have the following theorem.

THeORem 3.1. There is a $\underline{\lambda}$ such that (1.4) has no positive solution for $\lambda<\underline{\lambda}$.

For the proof, we need some knowledge on the following eigenvalue problem:

$$
\begin{array}{cc}
-\Delta_{p} u+|u|^{p-2} u=0 & \text { in } \Omega, \\
|\nabla u|^{p-2} \frac{\partial u}{\partial \nu}=\lambda|u|^{p-2} u & \text { on } \partial \Omega .
\end{array}
$$

This problem was studied in $[4,6]$ (see also [5]). It was proved there that problem (3.2) has a first positive eigenvalue $\lambda_{1}$ given by

$$
\lambda_{1}=\min _{u \in W^{1, p}(\Omega) \backslash W_{0}^{1, p}(\Omega)} \frac{\int_{\Omega}|\nabla u|^{p}+|u|^{p} d x}{\int_{\partial \Omega}|u|^{p} d \sigma},
$$

where $d \sigma$ is the boundary measure. In the linear case, $p=2$, problem (3.2) is known as the Steklov problem (see [1]).

Proof of Theorem 3.1. If (1.4) has a positive solution $u$, multiplying (1.4) by $u$, integrating by parts, and using (3.1) gives

$$
\int_{\Omega}|\nabla u|^{p}+|u|^{p} d x=\lambda \int_{\partial \Omega} f(x, u) u d \sigma \leq C \lambda \int_{\partial \Omega}|u|^{p} d \sigma
$$

and hence $\lambda \geq \lambda_{1} / C$ by (3.3), proving Theorem 3.1.

Now we prove the multiplicity result.

The assumptions in this case are as follows: let $G(x, t)=\int_{0}^{t} g(x, s) d s$, and assume the following:

$\left(\mathrm{G}_{1}\right)$ there exists $\delta>0$ such that $G(x, t) \leq 0$ for $0 \leq t \leq \delta$,

$\left(\mathrm{G}_{2}\right)$ there exists $t_{0}>0$ such that $G\left(x, t_{0}\right)>0$,

$\left(\mathrm{G}_{3}\right) \limsup \sup _{t \rightarrow \infty} G(x, t) / t^{p} \leq 0$ uniformly in $x$.

Theorem 3.2. Assume (3.1) and $\left(G_{1}\right),\left(G_{2}\right)$, and $\left(G_{3}\right)$ hold. Then, there is a $\bar{\lambda}$ such that (1.4) has at least two positive solutions $u_{1}>u_{2}$ for $\lambda \geq \bar{\lambda}$.

Observe that for problem (1.4) we can prove that the two solutions are ordered. We believe that this should hold also for (1.3), but the truncation argument used in the proof does not work because it destroys the variational structure of (1.3).

Again, set $g(x, t)=0$ for $t<0$, and consider the $C^{1}$ functional

$$
\mathscr{G}_{\lambda}(u)=\frac{1}{p} \int_{\Omega}|\nabla u|^{p}+|u|^{p} d x-\lambda \int_{\partial \Omega} G(x, u) d \sigma, \quad u \in W^{1, p}(\Omega) .
$$


Arguing as before, if $u$ is a critical point of $\mathscr{G}_{\lambda}$, denoting by $u^{-}$the negative part of $u$,

$$
\begin{aligned}
0 & =\left(\mathscr{G}_{\lambda}^{\prime}(u), u^{-}\right) \\
& =\int_{\Omega}|\nabla u|^{p-2} \nabla u \cdot \nabla u^{-}+|u|^{p-2} u u^{-} d x-\lambda \int_{\partial \Omega} g(x, u) u^{-} d \sigma \\
& =\left\|u^{-}\right\|_{W^{1, p}(\Omega)}^{p},
\end{aligned}
$$

hence we have that $u \geq 0$. Furthermore, by [10], $u \in C^{1, \alpha}(\Omega)$ and so, by the strong maximum principle and Hopf's lemma (see [12]), it follows that either $u>0$ in $\bar{\Omega}$ or $u \equiv 0$. Therefore, nontrivial critical points of $\mathscr{F}_{\lambda}$ are positive solutions of (1.4). Observe that in this case, the solution $u$ is positive up to the boundary.

By $\left(\mathrm{G}_{3}\right)$ and (3.1), there is a constant $C_{\lambda}>0$ such that

$$
\lambda G(x, t) \leq \frac{\lambda_{1}}{2 p}|t|^{p}+C_{\lambda}
$$

and hence

$$
\begin{aligned}
\mathscr{G}_{\lambda}(u) & \geq \frac{1}{p} \int_{\Omega}|\nabla u|^{p}+|u|^{p} d x-\int_{\partial \Omega} \frac{\lambda_{1}}{2 p}|u|^{p}+C_{\lambda} d \sigma \\
& \geq \frac{1}{2 p}\|u\|_{W^{1, p}(\Omega)}^{p}-C_{\lambda}|\partial \Omega|_{N-1},
\end{aligned}
$$

so $\mathscr{G}_{\lambda}$ is bounded from below and coercive.

Therefore, as $\mathscr{G}_{\lambda}$ is weakly lower semicontinuous, we obtain a global minimizer $u_{1}$. Once again, if $\lambda$ is big enough, this minimizer is nontrivial.

Lemma 3.3. There is a $\bar{\lambda}$ such that $\inf \mathscr{G}_{\lambda}<0$, and hence $u_{1} \neq 0$, for $\lambda \geq \bar{\lambda}$.

Proof. Take the constant function $u_{0} \equiv t_{0}$, where $t_{0}$ is as in $\left(\mathrm{G}_{2}\right)$.

Then, we obtain

$$
\int_{\partial \Omega} G\left(x, u_{0}\right) d \sigma=\int_{\partial \Omega} G\left(x, t_{0}\right) d \sigma>0
$$

Hence, $\mathscr{G}_{\lambda}\left(u_{0}\right)<0$ for $\lambda$ large enough.

The main difference in the arguments arrives at this point. As we mentioned before, by a truncation argument we can prove that the two solutions are ordered. In fact, fix $\lambda \geq \bar{\lambda}$. Let

$$
\tilde{g}(x, t)=\left\{\begin{array}{ll}
g(x, t), & t \leq u_{1}(x), \\
g\left(x, u_{1}(x)\right), & t>u_{1}(x),
\end{array} \quad \tilde{G}(x, t)=\int_{0}^{t} \tilde{g}(x, s) d s .\right.
$$

Then consider

$$
\tilde{G}_{\lambda}(u)=\frac{1}{p} \int_{\Omega}|\nabla u|^{p}+|u|^{p} d x-\lambda \int_{\partial \Omega} \widetilde{G}(x, u) d \sigma .
$$


1054 Quasilinear problems with sign-changing nonlinearities

If $u$ is a critical point of $\tilde{\mathscr{G}}_{\lambda}$, then $u \geq 0$ as before. Now,

$$
\begin{aligned}
0= & \left(\tilde{\varphi}_{\lambda}^{\prime}(u)-\varphi_{\lambda}^{\prime}\left(u_{1}\right),\left(u-u_{1}\right)^{+}\right) \\
= & \int_{\Omega}\left[\left(|\nabla u|^{p-2} \nabla u-\left|\nabla u_{1}\right|^{p-2} \nabla u_{1}\right) \cdot \nabla\left(u-u_{1}\right)^{+}\right. \\
& \left.\quad+\left(|u|^{p-2} u-\left|u_{1}\right|^{p-2} u_{1}\right)\left(u-u_{1}\right)^{+}\right] d x \\
& \quad-\lambda \int_{\partial \Omega}\left(\tilde{g}(x, u)-g\left(x, u_{1}\right)\right)\left(u-u_{1}\right)^{+} d \sigma \\
= & \int_{\left\{u>u_{1}\right\}}\left[\left(|\nabla u|^{p-2} \nabla u-\left|\nabla u_{1}\right|^{p-2} \nabla u_{1}\right) \cdot\left(\nabla u-\nabla u_{1}\right)\right. \\
& \left.\quad+\left(|u|^{p-2} u-\left|u_{1}\right|^{p-2} u_{1}\right)\left(u-u_{1}\right)^{+}\right] d x \\
\geq & \int_{\left\{u>u_{1}\right\}}\left[\left(|\nabla u|^{p-1}-\left|\nabla u_{1}\right|^{p-1}\right)\left(|\nabla u|-\left|\nabla u_{1}\right|\right)\right. \\
& \left.\quad+\left(|u|^{p-1}-\left|u_{1}\right|^{p-1}\right)\left(|u|-\left|u_{1}\right|\right)\right] d x \geq 0,
\end{aligned}
$$

so $u \leq u_{1}$. Therefore, $u$ is a solution of (1.4).

Now, as in the previous case, we will obtain the second solution as a critical point of $\tilde{\mathscr{G}}, u_{2}$, with $\tilde{\mathscr{G}}_{\lambda}\left(u_{2}\right)>0$ via the mountain pass lemma, which would complete the proof since $\tilde{\mathscr{G}}_{\lambda}(0)=0>\widetilde{\mathscr{G}}_{\lambda}\left(u_{1}\right)$.

LEMMA 3.4. The origin is a strict local minimizer of $\tilde{G}_{\lambda}$.

Proof. Let $\Gamma_{u}=\left\{x \in \partial \Omega: u(x)>\min \left\{u_{1}(x), \delta\right\}\right\}$. By (3.10) and $\left(\mathrm{G}_{1}\right), \widetilde{G}(x, u(x)) \leq 0$ on $\partial \Omega \backslash \Gamma_{u}$, so

$$
\tilde{\mathscr{G}}_{\lambda}(u) \geq \frac{1}{p}\|u\|_{W^{1, p}(\Omega)}^{p}-\lambda \int_{\Gamma_{u}} \tilde{G}(x, u) d \sigma
$$

By (3.1), Hölder's inequality, and Sobolev trace theorem,

$$
\int_{\Gamma_{u}} \widetilde{G}(x, u) d \sigma \leq C \int_{\Gamma_{u}} u^{p} d \sigma \leq C\left|\Gamma_{u}\right|_{N-1}^{1-p / q}\|u\|_{W^{1, p}(\Omega)}^{p},
$$

where $q=(N-1) p /(N-p)$ if $p<N$ and $q>p$ if $p \geq N$, so in order to finish the proof we need to show that $\left|\Gamma_{u}\right|_{N-1} \rightarrow 0$ as $\|u\|_{W^{1, p}(\Omega)} \rightarrow 0$.

Let $k=\min \left\{\min _{\partial \Omega} u_{1} ; \delta\right\}$, where $\delta$ is given in $\left(\mathrm{G}_{1}\right)$. Then,

$$
\|u\|_{W^{1, p}(\Omega)}^{p} \geq C \int_{\partial \Omega} u^{p} d \sigma \geq C \int_{\Gamma_{u}} u^{p} d \sigma \geq C k^{p}\left|\Gamma_{u}\right|_{N-1}
$$

as we wanted to show.

Now, we are in position to finish the proof of Theorem 3.2. 
Proof of Theorem 3.2. The same argument used for $\mathscr{G}_{\lambda}$ shows that $\tilde{G}_{\lambda}$ is also coercive, so every Palais-Smale sequence of $\widetilde{\mathscr{G}}_{\lambda}$ is bounded and hence contains a convergent subsequence as usual. Now, the mountain pass lemma gives a critical point $u_{2}$ of $\tilde{\mathscr{G}}_{\lambda}$ at the level

$$
c:=\inf _{\gamma \in \Gamma} \max _{u \in \gamma([0,1])} \tilde{G}_{\lambda}(u)>0
$$

where $\Gamma=\left\{\gamma \in C\left([0,1], W^{1, p}(\Omega)\right): \gamma(0)=0, \gamma(1)=u_{1}\right\}$ is the class of paths joining the origin to $u_{1}$.

\section{Acknowledgment}

This paper was partially supported by ANPCyT PICT 03-05009 and 03-10608, CONICET PIP0660/98 and PEI6388/04, UBA X052 and X066 and Fundación Antorchas 13900-5. J. Fernández Bonder is a member of CONICET.

\section{References}

[1] I. Babuška and J. Osborn, Eigenvalue problems, Handbook of Numerical Analysis, Vol. II, North-Holland, Amsterdam, 1991, pp. 641-787.

[2] L. Boccardo and D. G. de Figueiredo, Some remarks on a system of quasilinear elliptic equations, NoDEA Nonlinear Differential Equations Appl. 9 (2002), no. 3, 309-323.

[3] D. G. de Figueiredo and P. L. Felmer, On superquadratic elliptic systems, Trans. Amer. Math. Soc. 343 (1994), no. 1, 99-116.

[4] J. Fernández Bonder and J. D. Rossi, Existence results for the p-Laplacian with nonlinear boundary conditions, J. Math. Anal. Appl. 263 (2001), no. 1, 195-223.

[5] _ A nonlinear eigenvalue problem with indefinite weights related to the Sobolev trace embedding, Publ. Mat. 46 (2002), no. 1, 221-235.

[6] S. Martínez and J. D. Rossi, Isolation and simplicity for the first eigenvalue of the p-Laplacian with a nonlinear boundary condition, Abstr. Appl. Anal. 7 (2002), no. 5, 287-293.

[7] C. Maya and R. Shivaji, Multiple positive solutions for a class of semilinear elliptic boundary value problems, Nonlinear Anal. Ser. A: Theory Methods 38 (1999), no. 4, 497-504.

[8] K. Perera, Multiple positive solutions for a class of quasilinear elliptic boundary-value problems, Electron. J. Differential Equations 2003 (2003), no. 7, 1-5.

[9] P. H. Rabinowitz, Minimax Methods in Critical Point Theory with Applications to Differential Equations, CBMS Regional Conference Series in Mathematics, vol. 65, American Mathematical Society, Rhode Island, 1986.

[10] P. Tolksdorf, On the Dirichlet problem for quasilinear equations in domains with conical boundary points, Comm. Partial Differential Equations 8 (1983), no. 7, 773-817.

[11] N. S. Trudinger, On Harnack type inequalities and their application to quasilinear elliptic equations, Comm. Pure Appl. Math. 20 (1967), 721-747.

[12] J. L. Vázquez, A strong maximum principle for some quasilinear elliptic equations, Appl. Math. Optim. 12 (1984), no. 3, 191-202.

Julián Fernández Bonder: Departamento de Matemática, Facultad de Ciencias Exactas y Naturales, Universidad de Buenos Aires, 1428 Buenos Aires, Argentina

E-mail address: jfbonder@dm.uba.ar 


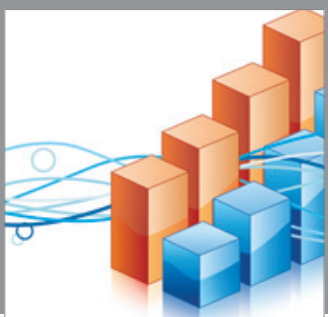

Advances in

Operations Research

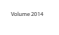

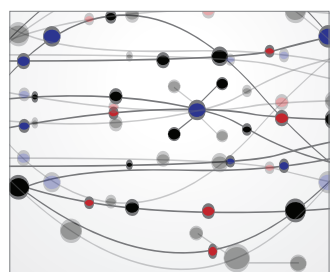

\section{The Scientific} World Journal
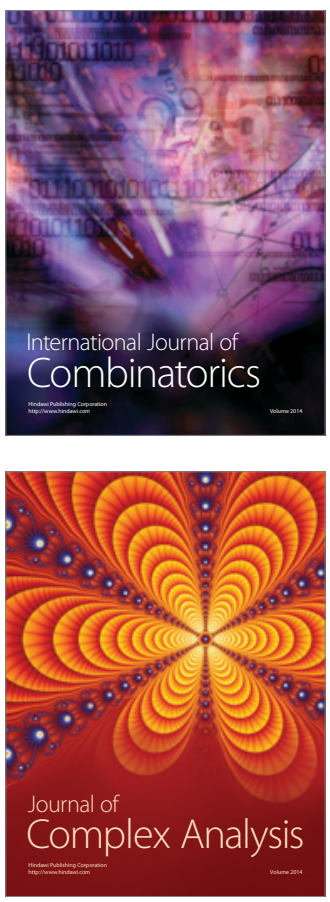

International Journal of

Mathematics and

Mathematical

Sciences
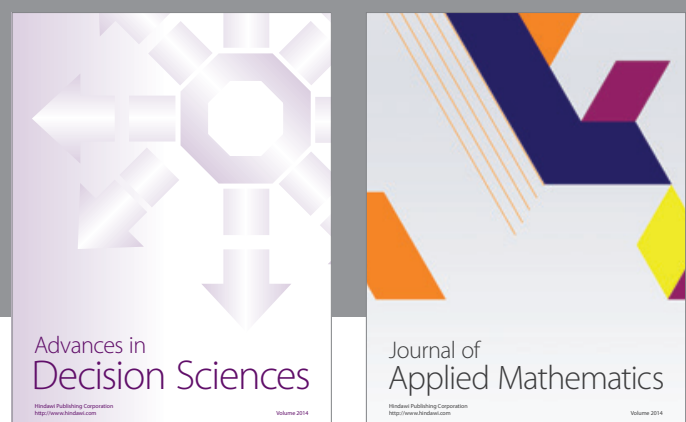

Journal of

Applied Mathematics
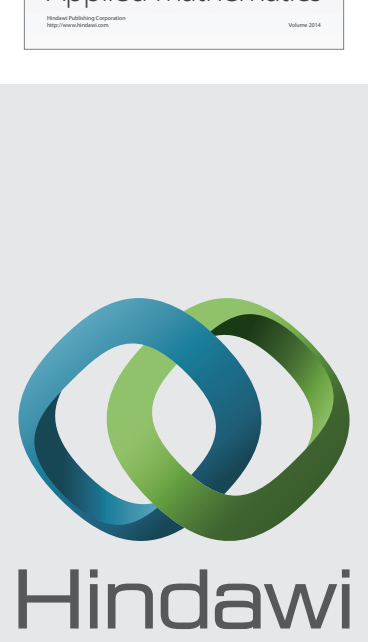

Submit your manuscripts at http://www.hindawi.com
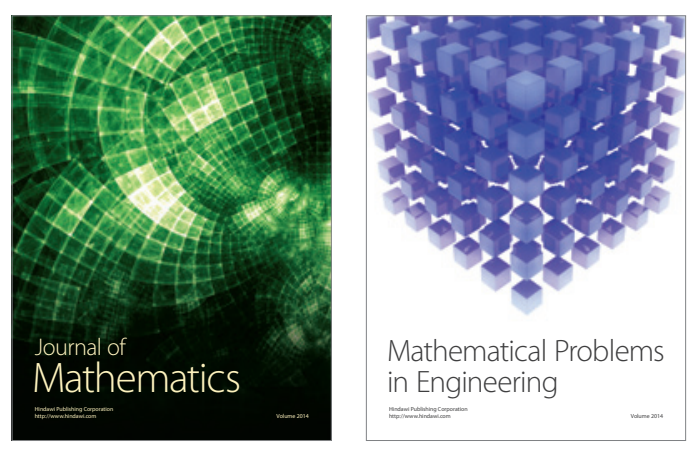

Mathematical Problems in Engineering
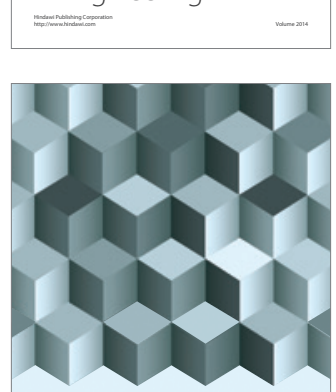

Journal of

Function Spaces
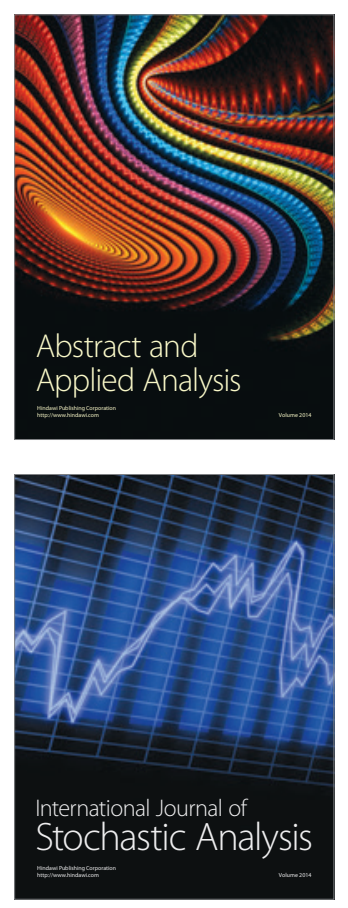

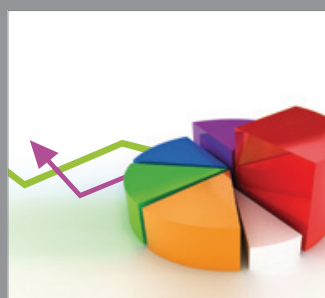

ournal of

Probability and Statistics

Promensencen
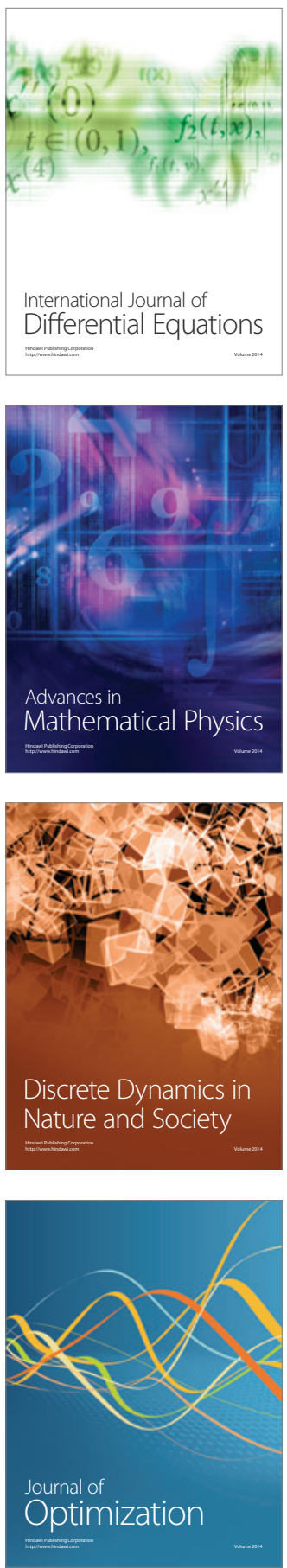